\title{
PAM or FLM in marine POM decomposition: microbial community succession in phylogenetic and functional diversity and capabilities with hydrostatic pressure
}

\author{
JIASONG FANG ${ }^{1}$, YING LIU ${ }^{1}$, LI ZHANG ${ }^{2}$ AND \\ MENGCHU ZENG ${ }^{3}$ \\ ${ }^{1}$ Shanghai Ocean University \\ ${ }^{2}$ Faculty of Earth Sciences, China University of Geosciences \\ ${ }^{3}$ China University of Geosciences \\ Presenting Author: jsfang@shou.edu.cn
}

Microbial-mediated decomposition of particulate organic matter (POM) during its downward transport from surface to the deep ocean constitutes a critical component of the ocean carbon cycle, but the extent to which hydrostatic pressure affects microbial succession and metabolic functions during POM decomposition is not fully known. In this study we investigated microbial community succession, shifting in phylogenetic and function diversity and metabolic capabilities during POM decomposition by particle-attached (PAM) and free-living microorganisms (FLM) subjected to increasing growth pressures. Diatom-originated ${ }^{13} \mathrm{C}$-labeled particulate organic matter was used to incubate microbial communities from the surface water of the East China Sea (ECS), at pressures of 0.1, 20, and $40 \mathrm{MPa}$ (megapascal). Our results showed that the PAM and FLM communities exhibited contrasting patterns and pressuredependencies in diversity, richness, and evenness. Microbial assembly was governed by deterministic processes for the in-situ communities and by stochastic processes for communities under high pressures. Co-occurrence network analysis revealed that the constructed networks of the total, PAM, and FLM communities exhibited scale-free, small world and modularity features. The PAM assemblage constituted module hubs of the community networks, whereas the FLM communities made up most of the connectors. Surprisingly, most of the identified module hubs and connectors were unclassified at the genus level and of low abundances, suggesting that our knowledge is limited about the potentially important ecological roles of rare taxa in the interacting communities of PAM and FLM. Metatranscriptome sequencing analysis revealed that expression of known metabolic pathways (carbohydrate, amino acid and energy production) varied greatly with pressure between PAM and FLM, indicating apparent difference in source utilization capacity that may prevent direct competition, but promote switching between microbes with different lifestyles and physiologies. Our results provide important insights into succession of microbial communities and metabolic functions associated with descending POM. 